\title{
Post-traumatic endophthalmitis caused by streptococcus species in preschool children: clinical features, antibiotic susceptibilities and outcomes
}

\author{
Yao Yang ${ }^{1} \cdot$ Wenjun Sui ${ }^{1} \cdot$ Fang Duan ${ }^{1} \cdot{\text { Yujie } \mathrm{Li}^{1} \cdot \text { Jieting Zeng }}^{1} \cdot$ Zhaoxin Jiang $^{1} \cdot$ Manli Liu ${ }^{1} \cdot$ Zhaohui Yuan $^{1} \cdot$ \\ Xiaofeng Lin $\mathbb{1}^{1}$
}

Received: 17 May 2020 / Revised: 4 January 2021 / Accepted: 27 January 2021 / Published online: 24 February 2021

(c) The Author(s) 2021. This article is published with open access

\begin{abstract}
Background/Objectives Streptococcus is a common cause of post-traumatic endophthalmitis in children. This study aimed to analyse the clinical features, antibiotic susceptibilities and outcomes of traumatic endophthalmitis caused by streptococcus in preschool children.

Subjects/Methods Patients aged $\leq 6$ years with traumatic streptococcal endophthalmitis seen at Zhongshan Ophthalmic Center between January 2013 and December 2018 were included in this retrospective study.

Results In total, 21 patients ( 21 eyes) were included. The mean age of the patients was $3.3 \pm 1.7$ years, where $57.1 \%$ were males. Scissors $(28.6 \%, n=6)$ were the most common cause of injury; $86.7 \%$ of patients were injured at home. Zone I $(80.9 \%)$ was the most common wound site; $90.5 \%$ of patients presented with a traumatic cataract. In general, Streptococcus pneumoniae (47.6\%) was the most common isolate. Viridans group streptococci accounted for $58.3 \%$ of cases in children aged 0-3 years, while $S$. pneumoniae accounted for $66.7 \%$ of cases in children aged $4-6$ years. The susceptibility rates of streptococcus to cefuroxime, levofloxacin and ofloxacin were $100 \%, 95.0 \%$ and $90.5 \%$, respectively. Although all the patients underwent vitrectomy combined with silicone oil tamponade, the final visual outcomes were no better than counting fingers.

Conclusions Although S. pneumoniae was the most prevalent organism in general, the dominant species varied between different age groups. The commonly used antibiotics, cefuroxime and fluoroquinolone, showed higher antibiotic susceptibility. Despite prompt treatment, the visual outcomes of paediatric post-traumatic endophthalmitis in preschool children were poor.
\end{abstract}

\section{Introduction}

Endophthalmitis is a rare but sight-threatening complication in patients with eye trauma [1,2]. Its incidence rate is estimated to be $2.8-54.2 \%$ in children with ocular trauma, varying geographically $[1,3,4]$.

These authors contributed equally: Yao Yang, Wenjun Sui

Zhaohui Yuan

yuanzhh@mail.sysu.edu.cn

$\triangle$ Xiaofeng Lin

linxiaof@mail.sysu.edu.cn

1 State Key Laboratory of Ophthalmology, Zhongshan Ophthalmic Center, Sun Yat-sen University, Guangzhou, China
The Streptococcus species is a common pathogen causing endophthalmitis. In adult-dominated populations, the streptococcus species is the leading cause of endophthalmitis after glaucoma filtering surgery. This species is also the common genus of organisms identified in endophthalmitis post cataract surgery and post intravitreal injection [5-8]. Among patients with streptococcal endophthalmitis, Streptococcus pneumoniae, viridans group streptococcus and group B $\beta$-haemolytic streptococcus were the main isolates, although the dominant isolates varied in different studies [8-11]. Previous studies have focused on paediatric post-traumatic endophthalmitis caused by all pathogens [12-14]. They found that the streptococcus species was a common cause of post-traumatic endophthalmitis in children. Thordsen et al. [2] reported that $29 \%$ of isolates were streptococcus in their study on paediatric post-traumatic 
endophthalmitis, and in another study, Al-Rashaed and Abu El-Asrar [15] found that streptococcus accounted for $53.7 \%$ of cases. Studies on adult-dominated streptococcal endophthalmitis found that despite prompt treatment, the visual outcomes were mostly poor, and about $24.7-33.3 \%$ of cases resulted in evisceration or enucleation $[9,11]$. However, there is insufficient information on streptococcal endophthalmitis in the paediatric population, hence it is necessary to conduct research on paediatric post-traumatic endophthalmitis caused by streptococcus.

This study retrospectively analysed the clinical characteristics, antibiotic susceptibilities and visual outcomes of post-traumatic streptococcal endophthalmitis in affected preschool children, providing valuable information on the clinical management of the disease.

\section{Subjects and methods}

\section{Population}

Consecutive medical records of all patients $\leq 6$ years old with culture-proven streptococcal traumatic endophthalmitis admitted to Zhongshan Ophthalmic Center, Guangzhou, in southern China, between January 2013 and December 2018, were retrospectively reviewed. This study was performed in compliance with the principles of the Declaration of Helsinki and was approved by the Institutional Ethics Committee of Zhongshan Ophthalmic Center, Sun Yat-sen University. The requirement for patient consent was waived, given the retrospective nature of the study.

\section{Procedures}

Personal information, including age and sex, the location where the injury occurred, the mechanism of injury and the duration from injury-to-primary repair were recorded. Each patient underwent ophthalmic examination conducted by an ophthalmologist. The characteristics of injury and endophthalmitis were obtained from the electronic medical record system. Data on medical treatment consisting of topical and intravenous use of antibiotics were recorded. Penicillin, cephalosporin, clindamycin and vancomycin were chosen for intravenous injection at the discretion of the ophthalmologist. Clindamycin was used when patients were allergic to cephalosporins. Surgical treatment, including intravitreal injection of vancomycin and pars plana vitrectomy combined with silicone oil tamponade, was performed based on the ophthalmologist's assessment. The average hospitalisation time and the average time from admission to vitrectomy were recorded. The visual acuity and silicone oil removal/non-removal were recorded at the last follow-up.

An aqueous/vitreous tap for culture was performed on the patients clinically diagnosed with endophthalmitis during surgery. The aqueous humour from the anterior chamber was aspirated through the corneal limbus with a needle attached to a 1-ml syringe. Vitreous specimens were collected through the pars plana prior to antibiotic injection or vitrectomy using a needle or vitrector. Samples were inoculated into bacterial and fungal media [16]. All bacterial isolates were subjected to species identification on the Vitek 2 Compact (BioMerieux, Inc., Marcy-l'Étoile, France). Antibiotic susceptibility testing of the isolated bacteria was performed using either minimal inhibitory concentration (MIC) methods or the Kirby-Bauer disc diffusion method, depending on the antibiotics. The antibiotic susceptibility was determined using the methods of the Clinical and Laboratory Standards Institute. The following antibiotics were tested in the susceptibility analysis: fluoroquinolones (levofloxacin and ofloxacin), cephalosporins (ceftazidime, cefazolin, cefoxitin and cefuroxime), aminoglycosides (tobramycin and neomycin), penicillin, azithromycin and chloramphenicol.

\section{Definitions}

The population was divided into two groups based on patients' age: 0-3 years and 4-6 years. The locations where the injury occurred were classified as either at home or in public, and the injury mode was classified as either self-injury or other-inflicted injury. The wound sites were categorised as zone I (wound limited to the corneal area, including the corneoscleral limbus), zone II ( $5 \mathrm{~mm}$ posterior to the corneoscleral limbus) and zone III (posterior to the anterior $5 \mathrm{~mm}$ of the cornea) [17]. Streptococci were further subdivided into $S$. pneumoniae, viridans group streptococci and S. pluranimalium. The cases were classified into concurrent endophthalmitis, which was defined as endophthalmitis diagnosed at presentation concurrently with an open globe, and post-open globe repair endophthalmitis, which was defined as eyes diagnosed with endophthalmitis after primary repair [18].

\section{Statistical analysis}

Statistical analysis was performed using SPSS version 16.0 (SPSS, Chicago, IL, USA). Characteristics of the study population and the isolates and the susceptibility rate were summarised using means and standard deviations for continuous variables and percentages for categorical variables. Differences in categorical variables were assessed using the Fisher exact test. Results with $P<0.05$ were considered statistically significant. 
Table 1 Management and visual outcome of paediatric streptococcal endophthalmitis by case.

\begin{tabular}{|c|c|c|c|c|c|c|c|c|c|c|}
\hline Case & Age & Sex & $\begin{array}{l}\text { Causes } \\
\text { of trauma }\end{array}$ & $\begin{array}{l}\text { Days from } \\
\text { injury to } \\
\text { primary repair }\end{array}$ & $\begin{array}{l}\text { Concurrent } \\
\text { endophthalmitis }\end{array}$ & $\begin{array}{l}\text { Presenting } \\
\text { visual acuity }\end{array}$ & $\begin{array}{l}\text { Intravenous } \\
\text { antibiotics }\end{array}$ & $\begin{array}{l}\text { Intravitreal } \\
\text { injection }\end{array}$ & $\begin{array}{l}\text { Vitrectomy } \\
+ \text { silicone oil }\end{array}$ & Final VA \\
\hline 1 & $8 \mathrm{~m}$ & M & Plant branch & 1 & Yes & - & Yes & No & Yes & - \\
\hline 2 & $1 \mathrm{y}$ & $\mathrm{F}$ & Metal wire & 3 & Yes & - & Yes & No & Yes & - \\
\hline 3 & $1 \mathrm{y}$ & $\mathrm{F}$ & Scissors & 0 & Yes & LP & Yes & No & Yes & $\mathbf{L P}$ \\
\hline 4 & $1 \mathrm{y}$ & $\mathrm{F}$ & Scissors & - & No & - & Yes & No & Yes & - \\
\hline 5 & $2 y$ & M & Uncertain & 2 & Yes & - & Yes & No & Yes & - \\
\hline 6 & $2 y$ & M & Plant branch & 2 & Yes & - & Yes & No & Yes & - \\
\hline 7 & $3 y$ & M & Metal nail & 8 & Yes & LP & Yes & Yes & Yes & - \\
\hline 8 & $3 y$ & M & Scissors & 0 & No & - & Yes & No & Yes & - \\
\hline 9 & $3 y$ & M & Toy & 0 & No & - & Yes & No & Yes & - \\
\hline 10 & $3 y$ & M & Bird & 4 & Yes & $\mathrm{HM}$ & Yes & Yes & Yes & LP \\
\hline 11 & $3 y$ & M & Scissors & 5 & Yes & - & Yes & Yes & Yes & - \\
\hline 12 & $3 y$ & $\mathrm{~F}$ & Plant branch & - & Yes & - & Yes & No & Yes & - \\
\hline 13 & $4 y$ & $\mathrm{~F}$ & Knife & Self sealed & Self sealed & $\mathrm{CF}$ & Yes & No & Yes & $\mathrm{HM}$ \\
\hline 14 & $4 y$ & M & Metal wire & Self sealed & Self sealed & - & Yes & No & Yes & LP \\
\hline 15 & $5 y$ & $\mathrm{~F}$ & Toy & Self sealed & Self sealed & $\mathrm{CF}$ & Yes & Yes & Yes & $\mathrm{CF}$ \\
\hline 16 & $5 y$ & $\mathrm{~F}$ & Scissor & 1 & Yes & - & Yes & Yes & Yes & HM \\
\hline 17 & $5 y$ & M & Metal stick & 0 & No & NLP & Yes & No & Yes & NLP \\
\hline 18 & $5 y$ & M & Scissors & 4 & Yes & NLP & Yes & Yes & Yes & $\mathrm{HM}$ \\
\hline 19 & $6 y$ & $\mathrm{~F}$ & Metal nail & 6 & Yes & NLP & Yes & Yes & Yes & - \\
\hline 20 & $6 y$ & M & Toy & 2 & No & - & Yes & No & Yes & LP \\
\hline 21 & $6 y$ & $\mathrm{~F}$ & $\begin{array}{l}\text { Injection } \\
\text { syringe }\end{array}$ & Self sealed & Self sealed & - & Yes & No & Yes & $\mathrm{HM}$ \\
\hline
\end{tabular}

\section{Results}

Twenty-one preschool children ( $\leq 6$ years old) (21 eyes) with streptococcal endophthalmitis after ocular trauma were enrolled in this study. The mean age of the patients was $3.3 \pm 1.7$ years (range: 8 months -6 years) and included 11 males $(57.1 \%)$. The demographic information, causes of injury, treatment measures and visual outcomes of participants are listed in Table 1.

Scissors $(28.6 \%, n=6)$ were the most common cause of injury, followed by toys and plant branches (both $14.3 \%$, $n=3$ ). Only one patient (4.8\%) presented with an intraocular foreign body. Besides the two patients whose records lacked primary repair information because of referral, four patients $(21.1 \%)$ presented with a self-sealed wound after injury and 15 patients $(78.9 \%)$ underwent wound repair. The average duration of injury-to-primary repair was $2.6 \pm$ 2.4 days. Among the patients, $57.1 \%$ were diagnosed with endophthalmitis concurrent with primary repair, and $23.8 \%$ of the patients developed endophthalmitis after primary repair. The average length of hospitalisation was $7.6 \pm$ 4.1 days. All patients received intravenous antibiotics: five received cephalosporin, three received cephalosporin combined with vancomycin, 11 received cephalosporin combined with clindamycin, one patient was treated with penicillin and one patient received clindamycin because of an allergy to cephalosporins. All patients underwent pars plana vitrectomy with silicone oil tamponade, and the average time from admission to vitrectomy was $1.8 \pm$ 2.5 days. Intravitreal vancomycin injection prior to vitrectomy was administered in seven patients (33.3\%). None of these patients developed panophthalmitis or orbital cellulitis, and none developed evisceration or enucleation due to uncontrollable infection.

The clinical characteristics are summarised in Table 2. Eighteen children $(85.7 \%)$ injured themselves and three (14.3\%) were injured by others. The medical records of 15 patients recorded the location of the injury, among whom $86.7 \%$ were injured at home. Zone I was the most common wound site $(80.9 \%)$, vitreous opacification $(95.2 \%)$, traumatic cataract $(90.5 \%)$ and hypopyon $(71.4 \%)$ were the top three common clinical symptoms. Retinal detachment occurred in $23.8 \%$ of patients.

Among the 21 patients, five patients $(23.8 \%)$ were lost to follow-up after discharge, and the average follow-up time was $12 \pm 14.4$ months (range: $1-53$ months). At the last follow-up, six patients (37.5\%) underwent silicone oil removal, and 10 patients were still maintained with silicone 
oil tamponade. Since most of the patients were young and unable to cooperate in visual acuity tests, only 10 patients had available visual acuity data in the last follow-up, and all the visual acuity results were no better than counting fingers.

The causative microorganisms are shown in Table 3 . Among the 21 isolates, $10(47.6 \%)$ were $S$. pneumoniae, nine $(42.9 \%)$ were viridans group streptococci and two $(9.5 \%)$ were $S$. pluranimalium. In the different age groups, viridans

Table 2 Injury aninformation d clinical characteristics of paediatric streptococcal endophthalmitis.

\begin{tabular}{lc}
\hline Variables & Findings \\
\hline Injury characteristics & \\
Location of injury* & \\
$\quad$ Home & $13 / 15(86.7 \%)$ \\
$\quad$ Public places & $2 / 15(13.3 \%)$ \\
Injury condition & \\
$\quad$ Injured by others & $3 / 21(14.3 \%)$ \\
$\quad$ Injured themselves & $18 / 21(85.7 \%)$ \\
Mean duration of injury-to- & $2.6 \pm 2.4$ day $\left(n=16^{*}\right.$, range: $\left.0-8\right)$ \\
primary repair & \\
Type of injury & \\
$\quad$ Penetrating & \\
$\quad$ Intraocular foreign & $20 / 21(95.2 \%)$ \\
body injury & $1 / 21(4.8 \%)$ \\
Time of diagnosis & \\
$\quad$ Concurrent endophthalmitis & $12 / 21(57.1 \%)$ \\
$\quad$ Post-OGR endophthalmitis & $5 / 21(23.8 \%)$ \\
$\quad$ Self-sealed & $4 / 21(19.1 \%)$ \\
Clinical characteristics & \\
Location of wound & \\
$\quad$ Zone I &
\end{tabular}

*Not all patients had available data for each variable. group streptococci were the most common isolates, accounting for $58.3 \%$ of cases in patients aged 0-3 years, but only $22.2 \%$ of cases in patients aged $4-6$ years $(p=0.18)$. The proportion of $S$. pneumoniae was as high as $66.7 \%$ in patients aged 4-6 years, but only $33.3 \%$ in patients aged $0-3$ years $(p=0.20)$.

The antibiotic susceptibilities of streptococcus in this study are summarised in Table 4. All the 21 isolates were sensitive to cefuroxime $(100 \%)$, and the susceptibility rate to ceftazidime and cefazolin reached $80 \%$. The susceptibility rate of streptococcus to chloramphenicol was $100 \%$, while that to levofloxacin and ofloxacin were $95.0 \%$ and $90.5 \%$, respectively. The susceptibility rate of streptococcus to penicillin was $62.5 \%$, and that of the aminoglycoside antibiotics such as neomycin, tobramycin and amikacin was as low as $30 \%(3 / 10), 19.0 \%(4 / 21)$ and $18.8 \%(3 / 16)$, respectively.

\section{Discussion}

In this study, we analysed the clinical data of 21 preschool children with post-traumatic endophthalmitis caused by streptococcus species in southern China. Our study showed a higher proportion of males than females, and S. pneumoniae was the most common isolate. However, the dominant isolates differed between the 0-3-year-old group and 4-6-year-old group. Streptococcus was sensitive to chloramphenicol, fluoroquinolone and cephalosporin. Despite aggressive treatment, the final visual acuity did not exceed counting fingers.

Previous studies on paediatric ocular trauma found that the proportion of males was close to that of females in younger children and increased with age [19-21]. Preschool patients aged 0-6 years were included in our study, and the proportion of males was close to that of females ( $57.1 \%$ vs. $42.9 \%$ ), consistent with the findings of previous studies. Gunes et al. reported that open globe injuries in preschool children ( $\leq 6$ years) occurred most frequently at home $(45.4 \%)$, and the most common cause of injury was kitchen items [22]. Similarly, our study found that over $85 \%$ of injuries occurred at home were mostly self-inflicted, and that scissors were the leading cause of injuries $(28.6 \%)$. Zone I was the most common wound entry site, and traumatic cataract
Table 3 The distribution of causative microorganisms confirmed by culture.

\begin{tabular}{lllrc}
\hline & Patients aged 0-3 years & Patients aged 4-6 years & Number & Percentage \\
\hline Streptococcus pneumoniae & $4 / 33.3 \%$ & $6 / 66.7 \%$ & 10 & $47.6 \%$ \\
viridans group streptococci & $7 / 58.3 \%$ & $2 / 22.2 \%$ & 9 & $42.9 \%$ \\
Streptococcus pluranimalium & $1 / 8.3 \%$ & $1 / 11.1 \%$ & 2 & $9.5 \%$ \\
Total & 12 & 9 & 21 & $100.0 \%$ \\
\hline
\end{tabular}


Table 4 Susceptibility rate of isolated bacteria to different antibiotics in paediatric endophthalmitis.

\begin{tabular}{|c|c|c|c|c|}
\hline & $\begin{array}{l}\text { Streptococcus } \\
\text { pneumoniae }\end{array}$ & $\begin{array}{l}\text { Streptococcus } \\
\text { viridans }\end{array}$ & $\begin{array}{l}\text { Streptococcus } \\
\text { pluranimalium }\end{array}$ & $\begin{array}{l}\text { Total } \\
\text { specimen }\end{array}$ \\
\hline \multirow[t]{2}{*}{ Levofloxacin } & $9 / 10$ & $8 / 8$ & $2 / 2$ & $19 / 20$ \\
\hline & $90.0 \%$ & $100.0 \%$ & $100.0 \%$ & $95.0 \%$ \\
\hline \multirow[t]{2}{*}{ Ofloxacin } & $9 / 10$ & $8 / 9$ & $2 / 2$ & $19 / 21$ \\
\hline & $90.0 \%$ & $88.9 \%$ & $100.0 \%$ & $90.5 \%$ \\
\hline \multirow[t]{2}{*}{ Ceftazidime } & $1 / 1$ & $2 / 2$ & $1 / 2$ & $4 / 5$ \\
\hline & $100.0 \%$ & $100.0 \%$ & $50.0 \%$ & $80.0 \%$ \\
\hline \multirow[t]{2}{*}{ Tobramycin } & $1 / 10$ & $1 / 9$ & $2 / 2$ & $4 / 21$ \\
\hline & $10.0 \%$ & $11.1 \%$ & $100.0 \%$ & $19.0 \%$ \\
\hline \multirow[t]{2}{*}{ Neomycin } & $1 / 4$ & $1 / 4$ & $1 / 2$ & $3 / 10$ \\
\hline & $25.0 \%$ & $25.0 \%$ & $50.0 \%$ & $30.0 \%$ \\
\hline \multirow[t]{2}{*}{ Cefuroxime } & $10 / 10$ & $9 / 9$ & $2 / 2$ & $21 / 21$ \\
\hline & $100.0 \%$ & $100.0 \%$ & $100.0 \%$ & $100.0 \%$ \\
\hline \multirow[t]{2}{*}{ Cefazolin } & $1 / 1$ & $2 / 2$ & $1 / 2$ & $4 / 5$ \\
\hline & $100.0 \%$ & $100.0 \%$ & $50.0 \%$ & $80.0 \%$ \\
\hline \multirow[t]{2}{*}{ Chloramphenicol } & $1 / 1$ & $2 / 2$ & $2 / 2$ & $5 / 5$ \\
\hline & $100.0 \%$ & $100.0 \%$ & $100.0 \%$ & $100.0 \%$ \\
\hline \multirow[t]{2}{*}{ Penicillin } & $6 / 9$ & $4 / 7$ & - & $10 / 16$ \\
\hline & $66.7 \%$ & $57.1 \%$ & - & $62.5 \%$ \\
\hline \multirow[t]{2}{*}{ Amikacin } & $1 / 9$ & $2 / 7$ & - & $3 / 16$ \\
\hline & $11.1 \%$ & $28.6 \%$ & - & $18.8 \%$ \\
\hline \multirow[t]{2}{*}{ Cefoxitin } & $4 / 9$ & $5 / 9$ & - & $9 / 18$ \\
\hline & $44.4 \%$ & $55.6 \%$ & - & $50.0 \%$ \\
\hline \multirow[t]{2}{*}{ Azithromycin } & $0 / 3$ & $1 / 2$ & - & $1 / 5$ \\
\hline & $0.0 \%$ & $50.0 \%$ & - & $20.0 \%$ \\
\hline
\end{tabular}

presented as the common sign in our study, consistent with previous studies on paediatric post-traumatic endophthalmitis $[1,21]$.

The most common isolates were varied in streptococcal endophthalmitis studies, where most participants were adults. Yospaiboon found that $S$. pneumoniae was dominant in 45 cases, while Kuriyan reported that viridans group streptococci were dominant in 63 cases $[8,11]$. Our study found that $S$. pneumoniae was more prevalent than viridans group streptococci in paediatric post-traumatic endophthalmitis $(47.6 \%$ vs. $42.9 \%$, respectively). We also found that the main isolates differed between the two age groups. Viridans group streptococci were the main isolates of posttraumatic streptococcal endophthalmitis in children aged 0-3 years, while $S$. pneumoniae was dominant in children aged 4-6 years. Viridans group streptococci are a heterogeneous group of organisms that can be human commensals, colonising the gastrointestinal and genitourinary tracts in addition to the oral mucosa [23]. Younger children, aged 0-3 years, were more likely to bite and scratch objects while playing, leading to viridans group streptococci infection through the mouth-object-eye route, which could explain the higher proportion of viridans group streptococci infection in this age group.
Vitrectomy can remove bacterial and inflammatory debris, preventing further damage. Previous studies found that vitrectomy combined with silicone oil tamponade could improve the anatomical and functional results in posttraumatic endophthalmitis in an adult-dominant population [24]. Jin et al. analysed the visual outcomes of posttraumatic endophthalmitis in patients aged $0-15$ years and found that, following combined vitrectomy and silicone oil tamponade, visual acuities were not only favourable but also often better than those predicted by Ocular Trauma Scores [25]. In our study, all the patients underwent vitrectomy combined with silicone oil tamponade, and the infection was successfully controlled with an average of 1.8 \pm 2.5 days from admission to surgery. However, the visual outcomes were unsatisfactory at the last follow-up. There are several reasons for this finding. First, streptococci are virulent bacteria that can cause severe inflammation. Although the viridans group streptococci are relatively lower in virulence compared with other streptococci, recent studies found that streptococcus species did not significantly influence the visual outcomes, and despite prompt treatment, most patients had poor outcomes [8, 9, 11]. Second, the patients included in our study were preschool children with an average age of $3.3 \pm 1.7$ years. The average 
duration of injury-to-primary repair was 2.6 days with $19.1 \%$ of patients presenting a self-sealed wound, and $57.1 \%$ of patients presenting typical signs of endophthalmitis at the time of primary repair. Young children may find it difficult to describe their symptoms clearly after injury, leading to delayed diagnosis and treatment of endophthalmitis, which might explain the poor visual outcomes despite prompt treatment upon admission [1, 15]. Previous studies on adult-dominated streptococcal endophthalmitis patients showed that $24.7-33.3 \%$ of cases resulted in evisceration or enucleation [8, 11]. In our study, infections were under control after vitrectomy combined with silicone oil tamponade, and there was no requirement for evisceration or enucleation during the follow-up period, even in patients who had vision with no light perception or those with slight atrophy of the eyeball.

Kuriyan et al. found that the susceptibility rates of streptococcus to ceftriaxone, vancomycin and levofloxacin were $98 \%, 95 \%$ and $93 \%$, respectively, in the adultdominated streptococcal endophthalmitis [8]. Jones et al. reported the drug sensitivity of $S$. pneumoniae in the United States and showed a sensitivity rate of $98.8 \%$ to levofloxacin and 99.1-100\% (susceptibility depending on breakpoint criteria) to ceftaroline [26]. In our study, streptococcus was sensitive to cephalosporins and fluoroquinolones, such as cefuroxime and levofloxacin $(100 \%$ and $95.0 \%$, respectively), consistent with the previous studies. S. pluranimalium is a species of streptococcus causing human infection reported in recent years [27]. Ganesh et al. recommended vancomycin, aminoglycoside and cephalosporin as the preferred antibiotics to treat $S$. pluranimalium infections [28]. Although only two isolates of $S$. pluranimalium were identified in our study, the two isolates were susceptible to levofloxacin, ofloxacin, tobramycin and cefuroxime. Therefore, to treat paediatric post-traumatic endophthalmitis caused by streptococcus, the topical use of antibiotics such as levofloxacin and cephalosporin eye drops are recommended. Considering the restriction on the systemic use of quinolones in children, cefuroxime is recommended for intravenous antibiotic treatment.

This study focused on the clinical features, antibiotic susceptibilities and outcomes of post-traumatic endophthalmitis caused by streptococcus in preschool children. However, there are also limitations arising from the retrospective nature of this study. The data, particularly the follow-up data, were incomplete. The patients in our study came from all over China, rendering the follow-up difficult because of distance. Young children hardly cooperated with the visual acuity test, especially in the best-corrected visual acuity test. In addition, because of the small sample size, some antibiotic susceptibilities were only tested in two strains in the subgroup, increasing the likelihood of bias.
In conclusion, we analysed the clinical characteristics, drug sensitivity and outcomes of post-traumatic streptococcal endophthalmitis in preschool children. S. pneumoniae was the most common isolate; however, viridans group streptococci were dominant in younger children aged 0-3 years. Both cefuroxime and levofloxacin showed excellent susceptibility and are thus recommended for treatment. Although the infection was under control after prompt treatment upon diagnosis, the vision outcomes were still poor.

\section{Summary}

\section{What was known before}

- Streptococcus species is the leading cause of endophthalmitis after glaucoma filtering surgery, and is also the common genus of organisms identified in endophthalmitis post cataract surgery and post intravitreal injection in adult-dominated population.

- Despite prompt treatment, the visual outcomes of streptococcal endophthalmitis in adult-dominated population were mostly poor, and about $24.7-33.3 \%$ of cases resulted in evisceration or enucleation.

- Streptococcus was a common cause of post-traumatic endophthalmitis in children.

\section{What this study adds}

- S. pneumoniae was the most common isolate in general; however, viridans group streptococci were dominant in younger children aged $0-3$ years.

- Both cefuroxime and levofloxacin demonstrated excellent susceptibility, and are thus recommended for treatment.

- Although the infection was under control after prompt treatment upon diagnosis, the vision outcomes were still poor.

Acknowledgements The authors would like to thank all staff involved in this study.

Funding This work was supported in part by funds from the National Natural Science Foundation of China (81900851), Natural Science Foundation of Guangdong Province, China (grant number: 2020A1515011316), Fundamental Research Funds of the State Key Laboratory of Ophthalmology (grant numbers: 30306020240020130 and 3030902113030) and High-level Hospital Construction Project (No. 303010401).

Author contributions YY, ZHY and XFL conceived and designed the study. WJS, YJL, MLL and JTZ acquired the data. YY, WJS, ZXJ and 
FD analysed and interpreted the data. YY, ZHY and XFL drafted the final manuscript.

\section{Compliance with ethical standards}

Conflict of interest The authors declare no competing interests.

Publisher's note Springer Nature remains neutral with regard to jurisdictional claims in published maps and institutional affiliations.

Open Access This article is licensed under a Creative Commons Attribution 4.0 International License, which permits use, sharing, adaptation, distribution and reproduction in any medium or format, as long as you give appropriate credit to the original author(s) and the source, provide a link to the Creative Commons license, and indicate if changes were made. The images or other third party material in this article are included in the article's Creative Commons license, unless indicated otherwise in a credit line to the material. If material is not included in the article's Creative Commons license and your intended use is not permitted by statutory regulation or exceeds the permitted use, you will need to obtain permission directly from the copyright holder. To view a copy of this license, visit http://creativecommons. org/licenses/by/4.0/.

\section{References}

1. Rishi E, Rishi P, Koundanya VV, Sahu C, Roy R, Bhende PS. Post-traumatic endophthalmitis in 143 eyes of children and adolescents from India. Eye (Lond). 2016;30:615-20.

2. Thordsen JE, Harris L, Hubbard GB 3rd. Pediatric endophthalmitis. A 10-year consecutive series. Retina. 2008;28:S3-7.

3. Lee CH, Lee L, Kao LY, Lin KK, Yang ML. Prognostic indicators of open globe injuries in children. Am J Emerg Med. 2009;27: 530-5.

4. Narang S, Gupta V, Simalandhi P, Gupta A, Raj S, Dogra MR. Paediatric open globe injuries. Visual outcome and risk factors for endophthalmitis. Indian J Ophthalmol. 2004;52:29-34.

5. Jacobs DJ, Leng T, Flynn HW Jr., Shi W, Miller D, Gedde SJ. Delayed-onset bleb-associated endophthalmitis: presentation and outcome by culture result. Clin Ophthalmol. 2011;5:739-44.

6. Wykoff CC, Parrott MB, Flynn HW Jr., Shi W, Miller D, Alfonso EC. Nosocomial acute-onset postoperative endophthalmitis at a university teaching hospital (2002-2009). Am J Ophthalmol. 2010;150:392-8 e392.

7. McCannel CA. Meta-analysis of endophthalmitis after intravitreal injection of anti-vascular endothelial growth factor agents: causative organisms and possible prevention strategies. Retina. 2011;31:654-61.

8. Kuriyan AE, Weiss KD, Flynn HW Jr., Smiddy WE, Berrocal AM, Albini TA, et al. Endophthalmitis caused by streptococcal species: clinical settings, microbiology, management, and outcomes. Am J Ophthalmol. 2014;157:774-80 e771.

9. Kurniawan ED, Rocke JR, Sandhu SS, Allen PJ. Predictors of visual outcome and the role of early vitrectomy in streptococcal endophthalmitis. Clin Exp Ophthalmol. 2018;46:424-31.

10. Mao LK, Flynn HW Jr., Miller D, Pflugfelder SC. Endophthalmitis caused by streptococcal species. Arch Ophthalmol. 1992; 110:798-801.
11. Yospaiboon Y, Meethongkam K, Sinawat S, Laovirojjanakul W, Ratanapakorn T, Sanguansak T, et al. Predictive factors in the treatment of streptococcal endophthalmitis. Clin Ophthalmol. 2018;12:859-64.

12. Bansal P, Venkatesh P, Sharma Y. Post-traumatic endophthalmitis in children: epidemiology, diagnosis, management, and prognosis. Semin Ophthalmol. 2018;33:284-92.

13. Sheng Y, Sun W, Gu Y, Grzybowski A. Pediatric posttraumatic endophthalmitis in China for twenty years. J Ophthalmol. 2017; 2017:5248767.

14. Yang Y, Lin L, Li Y, Jiang Z, Li C, Liu M, et al. Etiology, microbiological isolates, and antibiotic susceptibilities in cultureproven pediatric endophthalmitis: a 9-year review. Graefes Arch Clin Exp Ophthalmol. 2021;259:197-204.

15. Al-Rashaed SA, Abu El-Asrar AM. Exogenous endophthalmitis in pediatric age group. Ocul Immunol Inflamm. 2006;14:285-92.

16. Duan F, Wu K, Liao J, Zheng Y, Yuan Z, Tan J, et al. Causative microorganisms of infectious endophthalmitis: a 5-Year retrospective study. J Ophthalmol. 2016;2016:6764192.

17. Kuhn F, Morris R, Witherspoon CD. Birmingham eye trauma terminology (BETT): terminology and classification of mechanical eye injuries. Ophthalmol Clin North Am. 2002;15:139-43. v

18. Li X, Zarbin MA, Langer PD, Bhagat N. Posttraumatic endophthalmitis: an 18-Year Case Series. Retina. 2018;38:60-71.

19. Bucan K, Matas A, Lovric JM, Batistic D, Plestina Borjan I, Puljak L, et al. Epidemiology of ocular trauma in children requiring hospital admission: a 16-year retrospective cohort study. J Glob Health. 2017;7:010415.

20. Puodziuviene E, Jokubauskiene G, Vieversyte M, Asselineau K. A five-year retrospective study of the epidemiological characteristics and visual outcomes of pediatric ocular trauma. BMC Ophthalmol. 2018;18:10.

21. Yang Y, Yang C, Zhao R, Lin L, Duan F, Lou B, et al. Intraocular foreign body injury in children: clinical characteristics and factors associated with endophthalmitis. Br J Ophthalmol 2020;104:780-4.

22. Gunes A, Kalayc M, Genc O, Ozerturk Y. Characteristics of open globe injuries in preschool children. Pediatr Emerg Care. 2015;31: 701-3.

23. Doern $\mathrm{CD}$, Burnham CA. It's not easy being green: the viridans group streptococci, with a focus on pediatric clinical manifestations. J Clin Microbiol. 2010;48:3829-35.

24. Azad R, Ravi K, Talwar D, Rajpal, Kumar N. Pars plana vitrectomy with or without silicone oil endotamponade in posttraumatic endophthalmitis. Graefes Arch Clin Exp Ophthalmol. 2003;241:478-83.

25. Jin W, Xu Y, Wang W, Xing Y, Yang A. Efficacy and safety of 23-gauge pars plana vitrectomy/silicone oil tamponade combination for treatment of pediatric post-traumatic endophthalmitis. Curr Eye Res. 2017;42:1143-8.

26. Jones RN, Sader HS, Mendes RE, Flamm RK. Update on antimicrobial susceptibility trends among Streptococcus pneumoniae in the United States: report of ceftaroline activity from the SENTRY Antimicrobial Surveillance Program (1998-2011). Diagn Microbiol Infect Dis. 2013;75:107-9.

27. Aryasinghe L, Sabbar S, Kazim Y, Awan LM, Khan HK. Streptococcus pluranimalium: a novel human pathogen? Int J Surg Case Rep. 2014;5:1242-6.

28. Maher G, Beniwal M, Bahubali V, Biswas S, Bevinahalli N, Srinivas D, et al. Streptococcus pluranimalium: emerging Animal Streptococcal Species as Causative Agent of Human Brain Abscess. World Neurosurg. 2018;115:208-12. 